\title{
Takotsubo Cardiomyopathy linked with acute mania episode. A review.
}

$19^{\text {th }}$ WPA World Congress of Psychiatry

Lisbon, Portugal, 21-24 August, 2019

Batet Sánchez, D. Esparza Barandiaran, M. Hermosillo Torres, R. Pinilla Zulueta, R. Carnicer Castaño, Resident of Psichiatry, Resident of Clinical psychology.

- Objective: Takotsubo cardiomyopathy is a rare syndrome that is supposed to be linked with higher prevalences of anxiety and stress-related disorders. We observed one case of Takotsubo cardiomyopathy concurrent with acute mania exacerbation. Therefore, we examined it to further understand this cardiac's syndrome pathogenesis and its relationship to psychiatric illness.

- Background and aims: Takotsubo cardiomyopathy is a cardiac syndrome characterized by transient left ventricular dysfunction, electrocardiographic changes that can mimic acute myocardial infarction, and low-level release of myocardial enzymes in absence of obstructive coronary artery disease ${ }_{1}$. Has been found that $2 / 3$ patients suffering TTC carried a diagnosis of either anxiety or depression 2 . The psychiatric disorders may facilitate the syndrome via catecholamine mediation, triggering the damage and inducing the myocardial changes seen in the apical ballooning syndrome.

- Material and methods: We executed systematized research in Pubmed Platform using the key words "takotsubo syndrome" and "acute mania"; "takotsubo syndrome" and "bipolar disorder". The research showed twelve articles, two of them repeated. We excluded those not related to psychiatric conditions (4 articles) and published before 2010 (1 article). Following this inclusion and exclusion criteria, we have focused on five case-report articles.

- Results: Stress increases circulating catecholamines levels, resulting in more persistent physiological changes related to prolonged cardiac sympathetic stimulation. Throughout, catecholamine response to stressful situations and decreased inhibition of catecholamines is common in patients with psychiatric conditions. This catecholamines withdrawal is supposed to mediate the myocardial changes that have been seen in TCC.

- Conclusions: The influence of psychiatric illness in the pathogenesis of TTC may be an under-recognized association despite being reported in several times. We suggest that we should be aware of this condition and to increase the coordination between cardiologists and psychiatrists. Illness is often expressed in a vast spectrum of severity, and it's important to consider TTC as possible differential diagnoses.

Bibliography: (1) Maldonado JR, Pajouhi P, Witteles R. Broken Heart Syndrome (Takotsubo Cardiomyopathy) Triggered by Acute Mania: A Review and Case Report. Psychosomatics. 2013; 54(1):74-9. (2) Summers MR, Lennon RJ, Prasad A. Pre-morbid psychiatric and cardiovascular diseases in apical ballooning syndrome (takotsubo/stress-induced cardiomyopathy): potential predisposing factors?. J Am Coll Cardiol. 2010; 16;55(7):700-1. 